\title{
Trame di donne all'indomani della peste romana del 1656
}

\author{
La vicenda dell' "acqua tofana»
}

\author{
Simona FeCI
}

\author{
S. Feci, Università di Palermo, simona.feci@unipa.it
}

\begin{abstract}
Nel gennaio 1659 viene scoperta a Roma una rete di donne che produce, smercia e somministra veleno per sopprimere mariti. La rete è estesa, il numero delle vittime sembra ingente, la trama è in atto da almeno cinque anni e si è giovata del regime di eccezione prodotto dalla pestilenza che ha colpito la città (1656-1657). Il contributo intende esaminare il tema proposto dal punto di vista della storia delle donne: gli aspetti metodologici; un caso di studio che può inscriversi nella prospettiva di «all'indomani di»; gli effetti che il caso di studio scelto stesso ha ponendosi a sua volta come punto di svolta.
\end{abstract}

Roma, donne, peste, avvelenamento, giustizia

\begin{abstract}
Women's plots in the aftermath of the Roman plague of 1656. The "Acqua tofana" story. In January 1659 a network of women was discovered in Rome. They produce, sell, and administer poison to suppress husbands. The network is extensive, the number of victims seems huge, the plot has been in place for at least five years and has benefited from the exception regime produced by the pestilence that hit the city (1656-1657). The contribution intends to examine the theme from the point of view of women's history: the methodological aspects; a case study that can be inscribed in the aftermath perspective; the effects that the chosen case study itself has in turn set itself as a turning point.
\end{abstract}

Rome, women, plague, poisoning, justice

Il punto di partenza della riflessione sviluppata in queste pagine è il rapporto tra un avvenimento che introduce un radicale sovvertimento dell'ordine presente, ingenera un temporaneo vuoto e impone un nuovo regime istituzionale o di governo e le donne. La questione non è tanto includere la componente femminile nella storia generale. Sappiamo, infatti, che le donne partecipano insieme con gli uomini agli eventi che si verificano e ne sono coinvolte sia in quanto suddite di quegli ordinamenti ed esposte agli effetti delle vicende storiche, sia, benché meno spesso, in quanto autentiche protagoniste. Questo protagonismo, declinato in forme peculiari, è stato messo in luce da diverse analisi che, fin dagli anni Novanta, hanno sottolineato il ruolo attivo delle donne nelle sommosse e nelle rivolte popolari, la centralità rivestita in alcuni momenti-chiave delle rivoluzioni sei-settecentesche, il modo di interve- nire anche nei processi di costruzione delle nazioni e così via. E al tempo stesso hanno mostrato le ambiguità e gli stereotipi che le narrazioni storiche di quel protagonismo adottano e veicolano e che attengono alla partecipazione politica e all'esercizio di funzioni di governo, all'occupazione di spazi pubblici tipicamente maschili (come la piazza, il palazzo...), al rapporto con la violenza agita e in definitiva con il potere.

Riformuliamo allora la questione in modo più puntuale, interrogandoci su uno specifico effetto delle crisi e dei mutamenti politici che concerne le donne in quanto tali. In età contemporanea, i cambiamenti di ordine e di regime intervengono sulla condizione giuridica delle donne tracciando un'identificabile cronologia dei riconoscimenti e disconoscimenti dei diritti civili e politici, di cui le donne stesse sono le prime a fornirci l'attestazione e il conto. Per limitarci all'Italia, pensiamo solo alle 
istanze sollevate dalle donne durante le Repubbliche giacobine, quando si avanzano richieste di equiparazione e si lamentano le aspettative disattese; alle modifiche introdotte dai codici civili preunitari sul modello del Code francese o di quello austriaco; alla crisi verificatasi quando, con l'annessione del Lombardo-Veneto al Regno d'Italia, le donne di quei domini perdono l'autonomia riconosciuta alle coniugate dall'Allgemeines Bürgerliches Gesetzbuch (ABGB) per soggiacere all'autorizzazione maritale prevista dal Codice civile Pisanelli del 1865, sui cui caratteri e contenuti non mancano le critiche delle emancipazioniste; ancora, alla fine della prima guerra mondiale e poi all'indomani del secondo conflitto, quando le donne sono destinatarie di importanti innovazioni giuridiche (riconoscimenti di diritti o concessioni premiali) come l'abolizione dell'autorizzazione maritale nel 1919 e il diritto di voto nel 1946. Dal tardo Settecento, insomma, crisi e mutamenti di sistema investono direttamente le donne in quanto - se non altro - agiscono sulla loro condizione legale e le voci femminili sono, da quel momento in poi, in costante contrappunto con questi interventi.

Ma - e qui torniamo alla domanda - per l'età moderna possiamo proporre in modo plausibile un nesso tra il tempo del rivolgimento e una sua specifica proiezione sulla componente femminile della società che esso investe? Nella mia attività di ricerca, ho trovato almeno un caso che si può portare a esempio. Ho potuto verificare come solo all'indomani del Sacco del 1527 i notai romani cominciarono effettivamente ad applicare le disposizioni introdotte nel 1494 e riprese dagli statuti del 1521 e 1523 che imponevano alle contraenti una specifica procedura di stipula degli atti limitandone la capacità di agire. Le ragioni di questa dilazione e il collegamento con la cesura prodotta da un evento senza dubbio periodizzante restano di difficile decifrazione. Ma il mutamento è chiaramente chiaramente percepibile ${ }^{1}$. Sono rari gli accadimenti di questo genere e necessitano di ricerche approfondite, capaci forse di rileggere l'intero paradigma della storia politica e normativa.

1. Feci 2004.
VIRICIDIO E VELENO: FATTI E NARRAZIONI ATTORNO ALL' «ACQUA TOFANA» (1659)

La mia ricerca attuale intercetta il tema al centro di questa riflessione in modo tangenziale, ma pregnante. Nel gennaio 1659 venne scoperta a Roma una rete di produttrici e spacciatrici di un'acqua venefica, di cui erano acquirenti, secondo l'accusa, donne intenzionate a sopprimere i propri mariti. $\mathrm{Al}$ termine del processo, cinque di loro furono impiccate sulla piazza di Campo de' Fiori ai primi di luglio: Girolama Spana, Giovanna de Grandis e Maria Spinola, per la fabbricazione e la vendita del composto, Graziosa Farina e Laura Crispolti, solo per il commercio. Una sesta, Cecilia Verzellini, fuggita a Napoli e lì catturata ed estradata in autunno, fu giustiziata con la stessa modalità il $1^{\circ}$ marzo 1660 per aver somministrato la fatale mistura al genero. In quelle settimane di primavera, alcune mogli coinvolte nel processo dell'anno precedente furono condannate all'esilio dalla città o dallo Stato, alla detenzione domestica e ad altre pene. Tre viricide, invece, avevano ottenuto l'impunità in cambio di un resoconto dettagliato e veritiero delle azioni proprie e di coloro che, procurando il veleno, le avevano coadiuvate ${ }^{2}$. La vicenda è famosa, ma gli studi al riguardo rimontano agli anni ottanta dell'Ottocento ${ }^{3}$ e i riferimenti di epoca contemporanea non hanno aggiunto alcuna nuova informazione, anzi in alcuni casi hanno piuttosto fuorviato la ricostruzione con dati inattendibili o fantasiosi ${ }^{4}$.

Ho intrapreso quindi l'analisi dell'incartamento processuale e l'ispezione di fonti coeve che non erano ancora mai state consultate, oltre a rileggere quelle già note. Il lavoro di ricostruzione è delicato per il concorso di tipologie documentarie diverse, che vanno tenute distinte e, diversamente

2. ASR, Tribunale criminale del Governatore [da ora TCG], Processi, sec. XVII, b. 530; ivi, Registrazioni d'Atti, b. 308-31 1; le condanne a morte, anche in ASR, Inventario 285/II, Nomi dei giustiziati assistiti negli ultimi momenti dall'Arciconfraternita di S. Giovanni Decollato, già detta della Misericordia, ad nomen. Per l'uso di questo inventario: Di Sivo 2000, e p. 193 e 198, dove segnala anche l'assenza del registro relativo agli anni 1643-1665.

3. Ademollo 1881; Salamone-Marino 1881.

4. La ricordano, tra gli altri, Mari - Bertol 2001, p. 67-82; Messana 2007, p. 534-535; Fiume 2008; Pastore 2010, p. 204; Cavina 2011, p. 90-91; Muzzarelli 2013, p. 123124. Dal punto di vista letterario, merita ricordare Stendhal 1980, p. 239-240 (5 aprile 1828). 
da quanto operato finora, riportate e valutate nei rispettivi contesti di produzione: le carte giudiziarie, le cronache e le memorie posteriori al procedimento, la letteratura "di patibolo» e di consumo e, da ultimo, proprio gli ormai risalenti studi ottocenteschi (tralasciando in questa sede le opere di "fiction"). Per delineare i fatti quanto basta a introdurre gli aspetti che identificano i legami tra la vicenda dell' «acqua tofana» e la peste del 1656, conviene dichiarare subito che il caso è eccentrico rispetto al perimetro della criminalità femminile generica ed è fuori scala rispetto all'uso del veleno da parte di uomini o di donne che agiscono una tantum per liberarsi di coniugi o familiari: due campi di ricerca a cui la storiografia recente ha dedicato, e continua a dedicare, grande attenzione ${ }^{5}$.

Le indagini, infatti, coinvolgono oltre una ventina di donne tra imputate e testimoni, dislocate in cinque rioni della città (Parione, Monti, Trastevere, Borgo e Campo Marzio) e si concentrano su poco più di dieci presunte vittime, oltre ad alcune altre che, per la morte delle presunte responsabili o per ragioni di opportunità politica, non sono oggetto di approfondimento in sede processuale. Come vedremo, però, la risonanza e quindi la restituzione narrativa amplificano le dimensioni della mortalità inflitta dalle donne. Il veleno somministrato, cioè appunto l'acqua tofana, è un prodotto di facile preparazione domestica: arsenico e piombo, con una variante che prevede anche l'antimonio, polverizzati e posti a bollire in una pignatta di terracotta sigillata ${ }^{6}$. Il risultato è un liquido trasparente, insapore e inodore, che può essere facilmente mescolato in poche gocce a vino, brodo, minestra o altro alimento senza che la vittima avverta al gusto, all'olfatto e alla vista alcunché di sospetto. A seconda della concentra-

5. Sulla storia della criminalità femminile, Angelozzi Casanova 2014. Sulla relazione tra veleno e donne, rinvio alla precedente nt. 4 e, in una prospettiva d'oltralpe, a Bodiou - Chauvaud - Soria 2015.

6. ASR, TCG, Processi, sec. XVII, b. 530, c. 261r-v: «quando la detta Gerolama voleva far quell'acqua, pigliava dell'arsenico, che non so in quanta quantità si fosse, et pigliava del piombo limato, et li metteva dentro una pignatta et poi ci metteva dell'acqua di pozzo [...] che ci metteva un puoco d'antimonio et poi copriva la pignatta col coperchio et attornoattorno la sigillava il coperchio con la bocca della pignatta, con pasta fatta di farina di grano; et poi la metteva a bollire al fuogo un'hora, [...] poi la levava del fuogo, et poi raffreddata la levava dalla pignatta et votava l'acqua solamente dentro un fiasco o caraffa». zione della soluzione, l'effetto sarà più o meno tempestivo, ma in ogni caso le artefici consigliano una somministrazione misurata e graduale perché scongiura l'insorgere di dubbi intorno alla causa del decesso. L' «acquetta» non può essere neutralizzata da alcun rimedio che il medico, chiamato al capezzale della vittima che presenta sintomi di febbre e vomito e accusa dolori di stomaco, ordinerà per curare la presunta «malattia». Unico antidoto è l'ingestione di succo di limone o di altre sostanze acide.

La preparazione del veleno è intestata solo a due imputate: la palermitana Girolama Spana e la romana Giovanna de Grandis. L'una, dotata di maggiori conoscenze, sarebbe stata in grado di comporre anche filtri e prodotti per la cosmesi ${ }^{7}$; l'altra, invece, più limitata nel suo sapere. Entrambe le donne erano state istruite da una palermitana, tal Giulia, personaggio intorno alla quale si sono prodotte le maggiori distorsioni narrative. Attraverso di lei, infatti, si è stabilito il collegamento tra le protagoniste del caso romano e alcune donne che a Palermo, circa trent'anni prima, erano state condannate e giustiziate per la produzione e la somministrazione di acqua avvelenata. Giulia, infatti, sarebbe stata la presunta figlia di una di loro, Teofania d'Adamo, e la matrigna di Girolama Spana: il nome della mistura si legherebbe a Teofania, dalla quale anche Giulia avrebbe tratto l'appellativo di "Tofana» ${ }^{8}$. È proprio su Giulia, che si sono scatenate molte congetture prive di riscontri quando non del tutto infondate, come risulta dalle verifiche effettuate negli archivi romani e palermitani. Qui, però, basti dire che Giulia era morta diversi anni prima dei fatti contestati nel processo e pertanto è estranea all'arco cronologico che in queste pagine considero.

Il processo disegna un quadro compattamente al femminile, precipitando il lettore di quelle carte nel mondo delle donne della città: le loro biografie, la vita quotidiana, le attività economiche per il proprio sostentamento, le occupazioni giornaliere, i modi della coabitazione e la mobilità urbana, le devozioni, i conflitti e le crisi familiari, la socialità e le forme di solidarietà, la demografia attraverso i molteplici matrimoni e i numerosi parti, l'alfabetizzazione, le credenze... "Conforme si suole fare tra

7. Ivi, c. $340 \mathrm{v}-341$.

8. Ademollo 1881. 
noi altre donne» è un motivo costante che allude a parole, comportamenti e abitudini specifici - verosimili anche per il giudice, se non propriamente veritieri ${ }^{9}$. E, d'altronde, è solo attraverso una donna, infiltrata dalla corte per presentarsi come una potenziale cliente, che le autorità possono penetrare nel microcosmo delle avvelenatrici, coglierne in flagrante una e avviare la ramificata inchiesta giudiziaria ${ }^{10}$.

Dell'universo maschile a loro prossimo e familiare, le donne esaminate restituiscono - o direttamente rivolgendosial giudice o obliquamente narrando delle loro pratiche di conversazione e confidenza - un'immagine negativa: mariti assenti, perché hanno abbandonato la moglie, oppure, di contro, presenti in modo esorbitante, con pretese di comando e comportamenti violenti che esprimono forme di gelosia e possesso e una ancor più allarmante rapacità economica. D'altronde, sono proprio i coniugi i destinatari primi della mistura fatale, quelli in ogni caso su cui si appunta l'attenzione della corte, tanto che il reato delle donne è qualificato proprio in quanto atto compiuto ad effectum necandi eorum maritos. Ma non mancano nel catalogo delle presunte vittime anche generi, fratelli e nipoti prepotenti o scomodi ${ }^{11}$.

In questa narrazione femminile involontariamente corale, gli uomini sono lontani: entrano nel procedimento e prendono la parola in quanto magistrati e personale della corte, medici interpellati per rispondere del proprio intervento curativo sui deceduti, per formulare perizie sui campioni di acqua venefica ritrovata fortunosamente o per dare il parere sull'opportunità o meno di sottoporre a tortura alcune delle indagate, e testimoni de visu della malattia e del decesso delle vittime ${ }^{12}$. Soprattutto la «voce» degli uomini si leva successivamente al processo e detta la ricostruzione dell'accaduto che s'impone attraverso la cronaca

9. Ad esempio, ASR, TCG, Processi, sec. XVII, b. 530, c. 187r: «et la cominciai a conoscere dentro la chiesa della Minerva con l'occasione che io gli feci luogo di sedere nel banco dove io stavo nella chiesa dove io ero andata al Rosario et conforme si suol fare tra noi altre donne cominciassimo a discutere tra di noi et così stringessimo l'amicitia».

10. Ivi; Pallavicino 1839, p. 274.

11. Ad esempio Lorenzo Palazzi, figlio di Cecilia Gentile, presunta vittima della zia materna Elisabetta (ASR, TCG, Processi, sec. XVII, b. 530, c. 112).

12. Eccezione è la deposizione del solo marito di una delle spacciatrici del veleno, tutte altrimenti vedove. e la memoria del fatto e proprio il confronto, e in parte la divaricazione, tra risultanze emerse dal procedimento e la riproposizione dei fatti nelle narrazioni posteriori è sottesa alla questione affrontata del prossimo paragrafo.

\section{IL VELENO E LA PESTE DEL 1656-1657}

Il collegamento tra l'attività delle avvelenatrici e l'epidemia di peste che colpì Roma dal giugno 1656 all'agosto 1657 appare inevitabile. "Il contagio", infatti, è per le protagoniste della vicenda, e presumibilmente per molti altri romani, un evento periodizzante: distingue e data un prima e un dopo, oltre che un durante. Ed è anche il termine con cui si designa la peste ${ }^{13}$. Inoltre i fatti accertati dalla corte nel 1659 investono il quinquennio precedente, sebbene Girolama Spana e Giovanna de Grandis siano state messe a parte della composizione e del metodo di produzione dell'acqua al più tardi un decennio prima. La prima questione, dunque, sarebbe se, in occasione della peste, l'attività delle avvelenatrici si sia intensificata o meno. L'elevata moria di individui che accompagna le ondate dell'epidemia e la dimensione di straordinarietà che la pestilenza stabilisce consentono di confondere la mortalità ascrivibile alla volontà e alla mano umane entro quella ingenerata dal contagio. Una mimetizzazione che sconfina, per altro verso, nel richiamo delle cronache a fatti particolarmente eclatanti come il rianimarsi di individui ritenuti deceduti e in procinto di essere gettati nelle due fosse comuni predisposte fuori città o addirittura già ivi ${ }^{14}$.

Non solo. In alcune narrazioni posteriori a entrambi gli avvenimenti, la pestilenza e la trama delle avvelenatrici vengono collegate in modo diretto. Questa lettura retrospettiva fissata dai contemporanei solleva un secondo ordine di

13. Ad esempio: "Io mi sono recordata che dell'anno che cominciò [il] contaggio che non mi recordo il mese preciso ma credo che fisse del mese di aprile o di maggio, salvo il vero", ivi, c. $95 \mathrm{v}$; oppure "Tanto nel primo anno che cominciò il contagio quanto per tutto il tempo che durò habitai a casa di detto Andrea", ivi, c. 116; "Andrea morì nell'anno del contagio pare a me nel secondo anno in tempo d'estate avanti la raccolta dei grani», ivi, c. 382v; «un prelato che morì di contagio», ivi, c. 131v.

14. Roma-AC, Miscellanea 161/300; Rocciolo 2006, p. 130-134, edita la parte riguardante la pestilenza. 
problemi, che va al di là della concomitanza cronologica e attiene piuttosto proprio al modo in cui gli uomini e le donne di allora guardarono (e non guardarono) interpretarono (e non interpretarono) quei fatti e la loro cornice. L'analisi deve tener conto di due aspetti collegati nella lettura del «tempo di peste»: la correlazione tra peste e veleno, da un lato; la correlazione tra veleno e maleficio, dall'altro. I due nessi si dipanano all'interno di uno scenario che assume tratti peculiari nei diversi contesti secenteschi ma, alla luce degli studi condotti, appare caratterizzato nel complesso da elementi quali l'alterazione delle regole e dei criteri giuridici e sociali che presiedono alla ordinaria convivenza e la riformulazione di nuovi, la sospensione dei riti religiosi consueti e l'intervento di forme devozionali peculiari, l'interruzione almeno parziale dell'attività delle magistrature e l'imporsi di uno specifico ordinamento amministrativo e giurisdizionale che fissa e presidia spazi separati, reclusioni domestiche o ricoveri nei lazzaretti ${ }^{15}$.

Vediamo dunque i legami diretti tra i due avvenimenti. Il testo principale che sistematizza l'esperienza della pestilenza romana è il Tractatus de avertenda et profliganda peste politico-legalis di Girolamo Gastaldi, che era stato commissario generale dei Lazzaretti e poi commissario generale di Sanità per lo Stato della Chiesa e aveva diretto la gestione politico-sanitaria dell' epidemia ${ }^{16}$. Nell'opera, pubblicata nel 1684, Gastaldi riferisce che, all'epoca della pestilenza:

Disseminatum fuisse genus quoddam aquarum veneno infectarum, quarum auctores forminas fuisse, ex quxstione postmodum habita compertum est. Ea potione, quamplures dedita opera interfecti et coniugati prxcipue, quorum uxores aut eos è medio tollebant aut in causa erant, ut tollerentur. De his tamen omnibus supplicium sumptum ${ }^{17}$.

Nelle lettere superstiti tra quelle scritte durante la pestilenza (29 maggio-24 luglio 1657), Gastaldi non offre indicazioni al riguardo naturalmente, anche se non manca di segnalare le misure prese onde distinguere caso per caso le cause di morte e assegnare il singolo decesso alla pestilenza o ad altre ragioni. Ad esempio, di un «bichieraro» che esercitava alla Chiavica di S. Lucia, caduto morto in via Giulia, scrive: "pativa di vertigini dicono che havesse bevuto bene all'osteria... Si è fato ricognoscer, et anco aprire il cadavero, e li Medici non $c^{\prime}$ han trovato segno alcuno» ${ }^{18}$.

Ma il tempo del contagio induce confusione. Proprio una delle più controverse vicende riferite nel corso del processo alle avvelenatrici mostra l'intreccio tra i diversi piani. Il ventenne "giupponaro» Lorenzo Palazzi si ammala nel luglio 1656, mentre è già attiva la pestilenza. La febbre che accusa e il rumore delle carrette che trasportano i morti lo spaventano, secondo la madre Cecilia Gentili, e lo convincono, malgrado il parere contrario della donna, al ricovero all'Ospedale di S. Spirito, una volta ottenuta la patente di sanità da un medico. All'ospedale, dove peraltro in quegli anni era attivo il grande medico Paolo Zacchia ${ }^{19}$, Cecilia lo cura con un rimedio di acqua, zucchero e succo di limoncello suggeritole da un pittore, suo vicino di casa, con ottimi risultati. Al capezzale del malato però, giunge anche la zia, Elisabetta Gentili, con la quale il giovane è in contrasto. La donna, sprezzando il giudizio di Cecilia e il suo rimedio, dà al nipote uova crude, che gli suscitano vomito, dolori al petto e allo stomaco e costringono il barbiere dell'ospedale ad applicare un impiastro di triaca. D'altra parte, né il barbiere né il medico di S. Spirito sono in grado di formulare una diagnosi, mentre Lorenzo peggiora fino al decesso il $1^{\circ}$ agosto ${ }^{20}$. La malattia ordinaria, dunque, si accompagna alla pestilenza e conduce ad altri luoghi di ricovero e cura deputati. Ma la vicenda ha un seguito, che giustifica la sua esposizione nel corso del processo.

Di lì a poco, infatti, - sempre secondo la testimonianza di Cecilia - Elisabetta si lamenta con la sorella di non riuscire nell'impresa di ottenere la remissione di una querela per rissa sporta contro il suo proprio figlio, il macellaio Checco. Eppure, sostiene, in quei "tempi del contagio" sarebbe necessario perdonare ogni offesa ricevuta ed ella stessa chiede alla sorella di prometterle il suo perdono. Avuta la solenne rassicurazione, Elisabetta rivela a Cecilia: "sappi che io ho fatto morire tuo
15. Lo rilevano tutti gli studi sulla base dei provvedimenti emessi dalle autorità e dalle testimonianze: Fosi 2006.

16. Su Girolamo Gastaldi: Marsili 1999.

17. Gastaldi 1684, p. 116
18. Sonnino 2006, p. 71. lett. 26. L'intera serie di lettere è edita alle p. 64-74.

19. De Renzi 2008, p. 55-56.

20. ASR, TCG, Processi, sec. XVII, b. 530, c. $111 \mathrm{v}-113$ r. 
figliolo et l'ho fatto con quell'acqua che me diede quella donna che vedesti in casa", alludendo a una delle spacciatrici ${ }^{21}$. Il timore o l'approssimarsi della morte agiscono sugli individui ed Elisabetta, che «si sentiva male e dubitava di havere a morire presto", è spinta a confessare il misfatto. Cecilia, pur dichiarandosi addolorata nell'apprendere la causa della morte del figlio ancor più di quanto si fosse dispiaciuta al momento del decesso, perdona la sorella. Elisabetta, d'altronde, «il giorno seguente si amalò di contaggio et in tre giorni se ne morì, come anco morì l'istesso giorno detto Checco suo figliolo" ${ }^{22}$. La peste, che tra le vittime conta anche il barbiere e il medico dell'ospedale, non permette ai giudici di approfondire la fondatezza della vicenda oltre le parole di Cecilia, la quale nel 1660 sarà condannata all'esilio per il suo coinvolgimento nei traffici delle avvelenatrici. Nell'estate del 1657, sempre durante il contagio, muore anche un oste di Borgo, la cui moglie nemmeno due anni più tardi confesserà di averlo avvelenato, ottenendo tuttavia dal governatore l'impunità in cambio di rivelazioni che consentiranno di incriminare le autrici dell' «acquetta». Seguito il decesso dell'oste dopo una decina di giorni di allettamento e in presenza di sintomi via via più gravi (vomito, arsura, dolori di stomaco...), prima di comporre il corpo per la sepoltura «li medici [lo visitarono e appurarono] che non haveva segno alcuno di contagio ${ }^{23}$.

La sequenza individuo malato/defunto - non appestato - avvelenato ritorna in un altro caso eclatante. Gastaldi scrive in una lettera del 26 giugno 1657, a proposito di Francesco Maria Cesi, duca di Ceri:

È morto [...] con peteccie, e livore, e si dubita anco di tumore all'inguine, l'hò subito fato sapere a monsignor Montecatino, prelato del Rione, quale già ancor esso havea saputo la morte, e per accertarsi bene d'ogni cosa gli ho mandato à dire esser bene che si visiti il cadavero da medici, e chirurgi nostri ${ }^{24}$.

21. Ivi, c. $113 \mathrm{v}$.

22. Ivi, $114 \mathrm{r}$

23. Ivi, c. 385.

24. Sonnino 2006, p. 69, lett. 19, v. anche lett. 20. Francesco Maria Cesi, duca di Ceri e di Selci, marchese di Cantalupo, aveva sposato nel 1648 Maria Aldobrandini, figlia di Piero e Carlotta Savelli. Alla sua morte, il feudo fu ereditato dalla sorella Carlotta, moglie di Giovan Carlo Borromeo. La vedova, invece, fu protagonista negli anni immediatamente seguenti di una complessa relazione che la vide
Queste osservazioni sul decesso del duca e sull'accertamento delle cause hanno un contraltare nel processo del 1659. Qui, infatti, un'inquisita eccellente attribuisce la morte del nobiluomo all'acqua prodotta da Girolama Spana e fatta pervenire alla moglie Maria Aldobrandini. Racconta, dunque, Francesca Floris, al servizio di Giulia e Girolama Spana fin dai tempi della loro partenza da Palermo:

Quell'acqua che si fece dare il padre D. Gerolamo da Gerolama mia padrona per la duchessa di Ceri serviva e servì per il duca di Ceri suo marito perché io veddi doppo venire una cammeriera di detta signora duchessa [...] che parlò di segreto con detta mia padrona [...] E poi veddi che venne uno staffero a casa di detta Girolama a chiamarla due volte acciò che andasse dalla detta duchessa sicome ci andò, [...] e sentei tra tanto che il detto duca stava ammalato e dallì a pochi giorni sentij dire che era morto ${ }^{25}$.

Nella deposizione della serva, però, il nesso con la pestilenza è del tutto evaso per formulare invece un'accusa penale che, stando agli atti, non riceverà alcuna attenzione da parte della corte.

Resta il fatto che all'indomani e a seguito della scoperta delle avvelenatrici, il racconto del "contagio" include anche l'opera delle donne, in modo più o meno cospicuo. Gli Avvisi a stampa del 1659 , nel dare notizia della giustizia eseguita, indicano l'infittirsi dei venefici "massime nell'anno della peste $»^{26}$. La percezione compenetra anche altri testi. Abbiamo visto come Gastaldi dedichi loro una scarna ma eloquente menzione nel suo trattato. Invece l'Istoria stesa dall'abate Ruggero Caetani tra 1660 e 1666 si dilunga, proprio entro la trattazione della peste romana, sugli omicidi perpetrati dalle cinque giustiziate e sulla vastità della loro trama, per il numero dei decessi provocati così come per quello delle donne coinvolte. Annotava infatti il Caetani:

Ma è da sapersi, che non tutti morirono di contagio, poiché cinque maligne donne: Girolama, Giovanna, Betta, Anna e Caterina, unitesi in un paro di stanze, quasi in due caverne in un vicolo occulto laterale

unirsi coniugalmente con il conte, poeta e alchimista Francesco Maria Santinelli: Canneto 2017.

25. ASR, TCG, Processi, sec. XVII, b. 530, c. 264.

26. AAV, Segr. Stato, Avvisi, b. 32, c. 5 (5 luglio 1659). 
alla strada maestra della Longara, si provarono con le loro detestabili faccende di fare una compositione velenosa distillata con apparenza di acqua pura e con 5 [sic] gocce di quella mandavano un corpo sottoterra, e l'anima all'altro mondo. Onde conosciuto il male, ma non la provenienza del male, se il fisico ordinava l'emissione del sangue e che seguisse! non vi era più rimedio per il patiente! [...] Seppero così male operare queste cinque imprudenti, anzi perfide donne, che tra li 28mila morti vi è opinione che più di 7 mila ne morissero per havere ingoiato una presa di quell'acqua, data dalle cinque donne, e detta Acqua del Benvolere, poiché morendo alcuno per quella cagione non voleva più male al nemico. Ma poco doppo terminato lo scempio contagioso pagarono il fio de loro misfatti le 5 donne con una morte ignominiosa di forca in mezzo la piazza detta Campo de Fiore. Doppo di queste 5, se ne scoprirono altre molte complici, non solo che operavano e vendevano, ma circa 70 che havevano adoperato la detta acquetta per levarsi d'attorno i congionti ed i nemici (servendosi della congiontura della mala contagiosa influenza) ${ }^{27}$.

Il documento va sicuramente analizzato più in dettaglio per determinare le fonti delle notizie e la conformazione dei contenuti. Colpiscono, infatti, le indicazioni quantitative sulla moria di avvelenati, anche a fronte dei dati complessivi sui decessi del periodo di attività della pestilenza (che sappiamo essere stati nei quattordici mesi di contagio, circa 14.500, di cui solo 9.500 adducibili alla pestilenza $)^{28}$, il numero di donne coinvolte oltre quelle giustiziate, le informazioni dettagliate sulle sanzioni loro comminate. Appare molto difficile appurare se effettivamente le donne abbiano approfittato dell'accresciuta mortalità per procedere con gli omicidi. La serie di morti su cui insiste l'inchiesta rimonta al quinquennio precedente secondo un andamento ineguale: 1 (1654); 4 (1655); 1 (1656); 1 (1657); 4 (1658). Come mostra il caso di Lorenzo Palazzi, riferito ai magistrati durante il processo ma da costoro non indagato, la pestilenza inghiotte anche alcune donne che

27. Roma-AC, Miscellanea 161/300, c. 242v-243r.

28. Sonnino - Traina 1982, p. 440-441, alla luce di questi dati reputano "un grado di letalità $[. .$.$] incomparabilmente più$ basso di quello osservato negli stessi tempi e circostanze in altre città italiane», cioè l' $8 \%$ di popolazione deceduta rispetto al 50\% di Genova e Napoli, e un livello normale della restante quota di mortalità fisiologica. forse erano state acquirenti dell'acqua e artefici di soppressioni intenzionali di congiunti: vengono così a mancare al conto della giustizia criminale tanto le prime nel novero delle imputate, quanto i secondi tra le vittime ${ }^{29}$.

L'origine delle fonti e gli obiettivi sottesi all'estensione dei testi sembrano condizionare lo sviluppo della narrazione affidata a relazioni e cronache. Ce ne dà il senso la ricostruzione dell' «acqua tofana» condotta dal gesuita e cardinale Sforza Pallavicino nella Vita di Alessandro VII. La trattazione lunga e dettagliata della trama del veneficio, che egli definisce - con espressione fortunata - un "sordo macello dei mariti " $^{30}$, segue, senza sovrapposizione alcuna, l'esauriente resoconto della pestilenza, sebbene entrambe le vicende siano chiamate a testimoniare i successi della politica papale di fronte all'emergenza. Si può convenire che «la visibilità storica di un fenomeno come il crimine di avvelenamento è tributaria della visione che ne ebbero i contemporanei» ${ }^{31}$ : "visione» non del solo atto però, bensì di un più ampio quadro le cui coordinate sono, tra l'altro, la malattia e la cura e il contesto specifico. Durante la crisi epidemica, le autorità pubbliche riservarono una selettiva, prevalente attenzione ai decessi imputabili alla peste. La mimetizzazione delle morti procurate con quelle dovute alla pestilenza potrebbe essere stata agevolata dalla scelta preliminare compiuta dalle autorità romane: trattare ogni manifestazione morbosa come se fosse «peste» e attribuire ogni decesso repentino al contagio epidemico ${ }^{32}$. Fin dal giugno 1656, cioè quasi dall'inizio dell'epidemia, i parroci registrano nei libri dei defunti le cause della morte e specificano se esso si debba alla peste o meno ${ }^{33}$.

29. A proposito di una vedova, tal Lucia, acquirente di acqua da destinare al proprio fratello che le avrebbe impedito un nuovo matrimonio, si afferma: «La detta Lucia con tutti li suoi figlioli intesi dire poi che morissero tutti di contagio nel tempo del contagio qui in Roma l'anni passati, che io viddi la carretta sporca a casa loro quando andò a pigliare li figlioli per menarli al lazzaretto, che la madre era morta per prima», ASR, TCG, Processi, sec. XVII, b. 530, c. 203v; di alcune altre donne, potenziali testimoni perché presenti al decesso del primo marito, Caterina Giannotti dichiara la morte per il "contaggio", ivi, c. 276r.

30. Pallavicino 1837. Dalla Vita sarebbe stata estrapolata la Descrizione del contagio edita due anni più tardi (Pallavicino 1839); su questi testi: Favino 2014.

31. Muzzarelli, p. 117, che cita Collard.

32. Calvi 1990, p. 568

33. Rocciolo 2006, p. 115 
L'arco di tempo che la "malattia» degli avvelenati copre, dai primi sintomi al trapasso, si estende tra gli 8/9 giorni e le due settimane circa.

Inoltre, nell'infuriare della pestilenza, le autorità si preoccupano soprattutto di monitorare e reprimere in modo esemplare tutti i comportamenti elusivi le meticolose regole fissate negli editti della Congregazione di sanità per prevenire il diffondersi del contagio e puniscono con severità gli atti delittuosi direttamente conseguenti dalle disposizioni della normativa emergenziale come l'appropriazione e il commercio di abiti e masserizie appartenuti ad appestati, estorsioni ai danni di persone segregate per rilasciare loro bollettini di sanità falsi, abusi sessuali ${ }^{34}$. Gli studi rilevano l'esercizio di un forte e concertato controllo della crisi da parte delle istituzioni pontificie, che consentì - con le scelte politico-sanitarie compiute e la loro pronta e ferma esecuzione - di scongiurare anche episodi di follia collettiva come quelli clamorosi che segnarono la peste milanese del $1630^{35}$. A Roma, infatti, le autorità non indulsero - né consentirono che la popolazione indulgesse alla concezione della peste "manufatta", dunque al carattere artificiale e intenzionale della diffusione del contagio. A questo nesso alludono, invece, i riferimenti che identificano peste e veleno e leggono il contagio come "avvelenamento" o «veneficio». L'identificazione si presenta intanto a livello lessicale, attribuendo la denominazione di «veneficio» a tutte le pratiche di concorso intenzionale nella diffusione del contagio. A Torino, ad esempio, nel corso dell'ondata epidemica del 1630 si verificano diversi atti che, seppur il protomedico Giovanni Francesco Fiochetto denomina appunto «venefizij», sono identificabili piuttosto con la propagazione del contagio mediante le unzioni ${ }^{36}$.

Anche in occasione della pestilenza del 1656, il tema trova credito nella pubblicistica dell'epoca

34. Pastore 1991, p. 190-195; Calvi 1990, p. 562.

35. Pastore 1990, sia per il controllo dell'ordine pubblico nel regime eccezionale introdotto con la pestilenza, sia, p. 648, per la questione delle unzioni: "Meraviglia invece che in questo clima così favorevole al ruolo dell'immaginazione non si dia credito agli untori e all'opinione di una disseminazione diabolica ed artificiale del contagio: gli archivi criminali non ci aiutano in questa direzione, o al massimo possono offrire solo delle labili tracce che si possono collegare a quelle credenze».

36. Fiochetto 1631, p. 76-78. Per altri contesti, Pastore 1991, p. 71 n; Preto 1988. e si riverbera poi nelle fonti giudiziarie, soprattutto a Napoli e, in qualche misura, a Genova ${ }^{37}$. Nella città partenopea, durante la seconda metà di maggio del 1656, si verificarono tumulti contro presunti spargitori di polveri velenose propagatrici del contagio ed ebbero luogo esecuzioni capitali di particolare efferatezza. In generale, però, prevalsero le opinioni e soprattutto i comportamenti tesi a escludere il carattere diabolico o malefico del contagio. Il nunzio Giulio Spinola attribuì le dicerie sugli untori napoletani ai ministri regi, a tutela del modo in cui la crisi era fronteggiata:

Io però persisto a credere che [la diceria] sia invenzione per distrahere il popolo dal concetto formato che il male sia stato portato per colpa di chi soprintende ai negozi della sanità ${ }^{38}$.

Anche l'annalista genovese Filippo Casoni smentisce l'ipotesi della peste manufatta: gli esempi generalmente addotti, infatti, "mostrando l'intenzione e l'attentato, ma non l'esecuzione e la possibilità del fatto", corroborano proprio l'infondatezza di tale presunzione ${ }^{39}$. E complessivamente le teorie scientifiche circa l'origine e la diffusione del contagio e i metodi della profilassi "privata» pubblicate all'epoca, per quanto differenziate, non assegnano alla tesi della peste manufatta o magica alcuna significativa rilevanza ${ }^{40}$.

Sempre intorno ai nessi tra pestilenza e avvelenamenti, un'altra direzione di ricerca riguarda la disponibilità degli individui a ricercare e procurarsi rimedi efficaci che prevengano il contagio o curino la malattia. L'accesso socialmente differenziato ai medicamenti, che rianima «i fasti della farmacopea domestica e donnesca, e la fortuna di balsami e unguenti "segreti" (cioè di invenzione originale) dalle virtù molteplici, del tipo dell'“oglio balsamico perfettissimo" pubblicizzato in quei mesi nelle strade romane ${ }^{41}$, avrebbe forse consentito di propinare surrettiziamente anche $\mathrm{l}^{\prime}$ «acqua tofana». È allora, di nuovo, proprio la necessità di distinguere, in quello specifico contesto di pestilenza, la morte per malattia da quella per

37. Pastore 1990, p. 640-641; Calvi 1990, p. 561.

38. Cit. in Calvi 1981, p. 422.

39. Cit. in Preto 1988, p. 23.

40. Conforti 2006.

41. Donato 2006, p. 160. 
avvelenamento e, dunque, ricondurre il singolo decesso alla propagazione del contagio oppure all'intenzionale e subdola soppressione per mano umana che chiama in causa il lavoro di analisi e diagnosi dei medici. Il celebre Paolo Zacchia muore nel marzo 1659 e le sue Quaestiones medico-legales non conservano traccia della vicenda dell' «acqua tofana $»^{42}$. Tuttavia tra gli 85 pareri e perizie allegati all'opera uno riguarda il caso di un uomo, tal Tiberio Pantoschi, accusato di aver ucciso mediante il veleno la moglie Olimpia, morta invece di peste secondo la difesa e il consulto di parte ${ }^{43}$. La vicenda trattata dalla observatio risulta speculare a un'altra ricordata dall'abate Caetani proprio in coda al resoconto sulla pratica venefica delle cinque donne durante il contagio e a questa collegata.

Ne è protagonista un marito:

Il quale, essendo stufo della moglie, forse satio di pagarle il debito matrimoniale, comprò un'ampollina da Betta della sua acqua mortifera per il prezzo di 3 testoni e votandola da quell'ampolla la rovesciò entro una di quelle caraffine, ove suole stare la manna prodigiosa dell'Eroe di Bari, e si trovava haverla in pronto appunto, quando la consorte veniva gravata da alcuni fieri dolori, e per guarire dalli quali era stata consegliata prendere poche gocce di quella manna. Sentito ciò il buon marito subito corse a prendere quella caraffina, ove fuori di casa la teneva occulta, e portatala per darne un sorso alla consorte, la ritrovò assai migliorata, per esserseli scemati i dolori, onde non stimò bene forzarla a prendere quel medicamento per non insospettirla; e consegnata alla medesima l'ampollina confidentemente ne prese la cura di conservarla per i bisogni. Passati 15 giorni vennero i dolori al marito, e la innocente consorte, mentre quei stava nel colmo del contorcesi per gl'estremi dolori, le diede una presa de quella creduta manna, la quale parve che in quell'istante le facesse qualche operatione, con una picciola mitigatione de suoi acerbi dolori, ma intrinsecatasi cominciò a venirle la febretta ed in pochi giorni si venne all'emissione del sangue e crescendole il male, la povera consorte innocentemente le venne detto: "Oh Dio,

42. Per un inquadramento: Pastore - Rossi 2008; Pastore 2001.

43. Zacchia 1666, p. 122-124, consilium 77: Putredo cadaveris excludit praesumptionem propinati veneni: $\theta$ ex quibus dignosci possit quempiam ex veneno ingenito, vel externo periisse; Duffin 2011. non vorrei che in vece di giovare a suoi dolori, le havesse fatto male quella presa di manna che le diedi quando stava nel colmo dell'affanno del dolore!" All'ora il colpevole languente consorte, postosi in un dirotto pianto proruppe: "Ah consorte mia cara, tu mi hai ucciso innocentemente, et io moro avvelenato in pena dell'enorme errore, che commisi, quando comprai da Betta, con 3 testoni l'acquetta e la posi in quell'ampollina, ove era delineata l'effigie dell'Eroe della Manna di Bari, per dartene un sorso, e con quel credito liberarti da dolori, ma non essendo stato tale il mio pensiero ma di farti morire con una presa di quel maledetto liquore, il Cielo, che è giusto vindice di sì enorme fallo, mi punisce con la pena meritevolmente dovuta. Ti chiedo perdono, consorte mia cara, come altresi perdono a te la colpa innocente, per la quale meno vado a gl'Elisi". E ciò detto in poche ore, spirò irremissibilmente,

così come la stessa vedova avrebbe raccontato all'abate $^{44}$.

Alla luce di quanto osservato finora, possiamo chiederci se la scoperta della trama delle avvelenatrici, la sua percezione e definizione quale complotto di donne ai danni dei mariti, le dimensioni quantitative straordinarie (tanto più sconcertanti in quanto la fattispecie del veneficio è rara $)^{45}$, financo il motivo dell'acqua venefica e dell'avvelenamento liquido e quanto d'altro forgia la descrizione di quell'evento non veicolino un rigurgito dell'ansia e delle emozioni che pervadono l'esperienza della pestilenza a Roma. Qui, infatti, diversamente da quanto avviene altrove, le istituzioni esercitano un forte controllo e forse anche una repressione del sostrato emotivo che pervade l'esperienza collettiva e individuale del contagio. Questo sostrato però si dimostrerebbe capace di filtrare e di manifestarsi a distanza di

44. Roma-AC, Miscellanea 161/300, c. 243r-244r.

45. Tra il 1535 e il 1630 il tribunale criminale del Governatore celebrò 29 processi per veneficio, di cui solo 11, tutti entro 1544 e 1613, videro donne imputate del reato da sole (4 procedimenti) o insieme con uomini (7), senza peraltro che si configurassero come viricidi: Vasta 2015-2016, p. 250-259. Il libro dei condannati assistiti dalla confraternita romana di S. Giovanni Decollato riporta - oltre alla condanna delle donne coinvolte nei fatti del 1659 - solo un'altra avvelenatrice, giustiziata nel 1624: ASR, Inventario 285/II, Nomi dei giustiziati...: «1624, Giovanna, di Regno, impiccata a Ponte per aver dato veleno al marito». Per un confronto su questo aspetto con Bologna: Pastore 2010, p. 95 e 100-101. 
Trame di donne all'indomani della peste romana del 1656. La vicenda dell' «acqua tofana»

qualche tempo, retrospettivamente, agendo come rilettura del recente passato e come quadro entro cui leggere i fatti del presente. Vediamolo allora. La principale Relazione dei venefici esordisce:

Regnante papa Alessandro VII Chigi fel. mem. l'anno doppo la peste, si udì in Roma con stupore di tutti una specie di contaggio nuovo, mentre morivano più persone senza conoscersi e penetrarsi il male, e quello che rendeva maraviglia, era che non mutavano il colore nel volto anzi benche morti fossero, parevano vivi e che dormissero.

Lo stesso arrivo in Roma di Girolama Spana, secondo questa versione, sarebbe da collocare "dopo la mortalità cagionata dal morbo»" ${ }^{46}$. E la medesima associazione tra mortalità inflitta dalla peste e letalità dell'azione delle avvelenatrici torna anche nel principale testo "di consumo" su quei fatti, la Historia in 49 ottave di Francesco Ascione: «e le fabricatrici, e lor consorte, / smaltivano per Roma un tal lavoro, / che si potea ben dire con duolo interno / che peste fusse, anzi un contagio eterno» ${ }^{47}$.

E, d'altronde, mi sembra di poter stabilire una linea di continuità anche tra il modo in cui le autorità pubbliche affrontarono l'emergenza della pestilenza e come lo stesso papa, il governatore di Roma Giovan Francesco Baranzoni, il procuratore fiscale generale Giustino Gentili e i giudici perimetrarono e interpretarono la trama che si presentò a loro nel 1659. Il delitto contestato alle imputate, infatti, ha un profilo esclusivamente criminale, senza tentennamenti: de fabricatione aquae veneficae illiusque [...] dispensatione et venditione pluribus mulieribus ad effectum necandi eorum maritos ${ }^{48}$. Tale opzione va considerata alla luce della contaminazione che investe l'accusa di veneficio e quella di "fattucchieria», cioè di ricorso a pratiche di magia bianca, che la storiografia ha riscontrato essere molto frequente. La dimestichezza delle donne con

46. Bologna-BCA, ms A. 358, c. 662-685: Giustitie fatte nel Pontificato di Alessandro $7^{\circ}$ del 1659 di alcune vedove che fabricavano la velenosa bevanda dell'aquetta con la quale davano morte alli loro mariti, et altri, le quali furono fatte morire nella piazza di Campo de' Fiore; anche Roma-BC, ms 2355, Relatione dell'esemplar giustizia seguita nel Pontificato d'Alessandro Settimo nelle persone che fabricavano, e dispensavano l'acquetta, c. 275.

47. Ascione 1696. Sul testo: Baronti 2013, p. 202-203 e Baronti 2012. Salamone-Marino 1881 pubblica l'edizione napoletana del 1699

48. ASR, TCG, Registrazioni d'Atti, b. 308, c. 35v-36r. erbe e sostanze, la preparazione e l'erogazione di unguenti, polveri, impiastri, medicamenti destinati talora alla cerchia domestica, talora invece a un commercio per denaro, la concezione che presiede alla diagnosi e alla terapia in caso di malattia e il collegato ricorso a una pluralità di figure in grado di "guarire", ma anche una maggiore sensibilità dalla fine del XVI secolo per l'universo della stregoneria avrebbero potuto giocare un ruolo anche in questa vicenda così come avvenne ripetutamente in Sicilia ${ }^{49}$. E, infatti, sarebbe stato possibile, anche in presenza delle ammissioni di Giovanna de Grandis e di Maria Spinola, insistere e agire su alcune sottili tracce presenti nelle rivelazioni delle imputate e confermate dalle carte del S. Offizio. Giovanna, infatti, a suo dire, avrebbe attirato i sospetti del tribunale, sebbene la congregazione, pur trattenendola due mesi, non avesse riscontrato alcuna ragione neppure per una ancorché lieve sanzione $^{50}$. E Maria Spinola conoscerebbe segreti e filtri d'amore ${ }^{51}$. Sappiamo che il tribunale è percepito dai sudditi come istanza cui rivolgersi più per la posizione apicale che per le sue competenze in materia di fede ${ }^{52}$. E tuttavia, nel maggio 1656, Girolama Spana compare spontaneamente davanti al S. Officio e "se accusat de colloquijs factis circa futurum pontificem tempore sedis vacantis", cioè nel 1655, tra la morte di Innocenzo X e l'ascesa al soglio di Alessandro VII. Alcuni mesi dopo, all'inizio di agosto, Girolama, qualificata anche con il suo soprannome L'Indovina (richiamato anche nel processo del 1659 per la capacità di rinvenire oggetti perduti), è accusata da una certa Domenica Arcella e per questo motivo la congregazione dispone l'esame del testimoni informati e la perquisizione ${ }^{53}$. Proprio, nel corso di quell'anno, mentre infuria la pestilenza, le accuse di «sortilegio" sembrano moltiplicarsi

49. Leonardi 2005, p. 100-101; Messana 2007. Per le trasformazioni dalla fine del Cinquecento: Brambilla 2008.

50. ASR, TCG, Processi, sec. XVII, b. 530 , c. $32 \mathrm{v}$.

51. Ivi, c. 40r: «Et mi domandò se io haveo niente da far voler bene, et io gli dissi che non sapevo niente e perché la detta Giovanna mi dissi che quella donna cortegiana che era asieme con lei chiamata Margherita era stizzata con il suo homo [...] però era venuto a trovarmi perché io gli insegnasse qualche cosa per farli remettere insieme».

52. Ivi, c. 42: una donna a cui Giovanna aveva venduto per cinque scudi l'acqua velenosa per procurare la morte del marito, insoddisfatta del risultato si sarebbe recata a casa sua «a gridar lì dicendo che rivoleva li suoi quadrini che altrimenti la voleva andare ad acusare al S. ${ }^{\text {to }}$ Off. $^{\circ}{ }^{\prime}$.

53. ACDF, Decreta, 1656, c. 81 r e 110 r. 
coinvolgendo uomini e donne della città, diversamente da quanto accade negli anni seguenti quando le segnalazioni esaminate dalla congregazione risultano provenire soprattutto da sedi periferiche, e da Napoli in particolare ${ }^{54}$. Malgrado ciò, né il procedimento condotto davanti al tribunale criminale del Governatore (e seguito con la massima attenzione dal pontefice), né di contro i decreta del S. Officio lasciano trasparire una torsione dell'istruttoria verso temi e imputazioni diverse dal veneficio ${ }^{55}$. Il caso dell'«acqua tofana», dunque, si pone in stretta connessione con la pestilenza romana del 1656, nelle forme e nei termini che abbiamo visto.
In conclusione, tuttavia, dobbiamo osservare che la vicenda presenta anche delle aperture su scenari nuovi. Essa, infatti, concorre a ridefinire, nell'immediato e nei decenni successivi, i «regimi delle professioni»: all'indomani e in ragione dei fatti accaduti il governo papale ribadisce le regole riguardanti la circolazione di sostanze pericolose (come regolamento dell'accesso ai canali di approvvigionamento e dello smercio affidati ai farmacisti o speziali), accresce il controllo sulle cause dei decessi e perfeziona lo sviluppo delle tecniche investigative e istruttorie, coinvolgendo dunque farmacisti, medici e magistrati.

\section{Bibliografia}

\section{Archivi}

ACDF $=$ Archivio delle Congregazione per la Dottrina della Fede ASR $=$ Archivio di Stato di Roma.

$\mathrm{AAV}=$ Archivio Apostolico Vaticano.

Bologna-BCA = Bologna, Biblioteca comunale dell'Archiginnasio.

Roma-AC = Roma, Archivio Caetani.

Roma-BC $=$ Roma, Biblioteca Casanatense.

54. Nel 1656, da maggio a dicembre, i romani o residenti accusati di sortilegio sono: Giovanni barbiere romano (ivi, c. 76r); Lucia Moriconi (77r); Pietro Boschi alias Il Veneziano (indiziato per possesso di scritturesortileghe e detenuto nelle carceri di Tor di Nona, c. 82r); la palermitana Antonia Baroni e Onofria praetensas maleficas (82r); altri cinque uomini $(84 \mathrm{v}, 93,98 \mathrm{v}, 99 \mathrm{v}, 100 \mathrm{v})$; Ludovica alias Ludovicaccia e Narda, curiale, abitanti l'una vicino a S. Pietro in Vincoli, l'altra al vicolo de Babuccio (119rv); il pittore Pietro Colangeli, con la moglie Cinzia e la cognata Margherita Reda (124r); Caterina Anconitana tabaccaria in vicolo dei Verospi (128 e 131); la vedova Vittoria (134v); Bernardino de Fabijs sponte comparente (134v); il monaco benedettino Bernardo da Palermo per possesso di scripturas sortilegas (132v e 137v); Francesco Cocchi (139r); Anna Fedele, insieme con la figlia Caterina, la comare Agnese e il notaio del Governatore Stefano Magnano (148v-149; 154r). Solo due di costoro furono oggetto di misure ulteriori: Lucia Moriconi e Anna Fedele.

55. Non ho ancora potuto verificare la fondatezza dell'affermazione del diarista Gigli, secondo cui delle donne che con l'acqua venefica avevano procurato la morte di mariti e parenti, "ne furno molte murate nelle carcere delle Inquisitione» (Gigli 1994, II, 775). 
Trame di donne all'indomani della peste romana del 1656. La vicenda dell' "acqua tofana»

\section{Bibliografia primaria}

Ascione 1696 = F. Ascione, Historia del giusto castigo dato a cinque donne in Roma, le quali facevano, e dispensavano acque avvelenate, con le quale le donne davano morte à $i$ loro mariti. Giustitiate nella Piazza di Campo di Fiori [...], Ronciglione, Giacomo Menichelli, 1696 [ed. digitalizzata: http://www.fondazionemarcobesso.it/digilibro/ $\mathrm{bd} / \mathrm{ebooks} /$ reader/21].

Fiochetto 1631 = G.F. Fiochetto, Trattato della peste o sia contagio di Torino dell'anno 1630, Torino, Gio. Gugliemo Tisma, 1631.

Gastaldi $1684=$ G. Gastaldi, Tractatus de avertenda et profliganda peste politico-legalis, Bologna, Manolessi, 1684.

Gigli 1994 = G. Gigli, Diario romano, M. Barberito (ed.), Roma, Colomba, 1994.

Mirogli $1764=$ F. Mirogli, Istruzzioni teorico-prattiche criminali..., III, Roma, Salomoni, 1764.

Salomone-Marino 1905 = S. Salomone-Marino, La peste in Palermo negli anni 1624-1626. Relazione di anonimo, Palermo, Boccone, 1905.

Stendhal 1980 = Stendhal, Passeggiate romane, I, M. Colesanti (a cura di), Roma, Cassa di Risparmio di Roma, 1980.

Zacchia $1666=$ P. Zacchia, Quaestionum medico-legalium tomus tertius, Francoforte, Johannes Baptistae Schönwetteri, 1666.

Zacchia 2001 = P. Zacchia, Venefici dalle "Quaestiones medicolegales» di Paolo Zacchia, a cura di G. Pierini, Milano, 2001.

\section{Bibliografia secondaria}

Ademollo 1881 = A. Ademollo, I misteri dell'acqua tofana, Roma, 1881.

Angelozzi - Casanova 2014 = G. Angelozzi, C. Casanova, Donne criminali: il genere nella storia della giustizia, Bologna, 2014.

Baronti 2012 = G. Baronti, Donne e veleni. Conflitti di genere nella letteratura di piazza, in G. Baronti, D. Parbuono (a cura di), Studi di tradizioni popolari: passato e presente, Perugia, 2012, p. 35-71.

Baronti 2013 = G. Baronti, Storie funeste. Prudenza Anconitana e Marzia Basile: Francesco Novati e Benedetto Croce, Milano, 2013.

Bodiou-Chauvaud-Soria $2015=$ L. Bodiou, F. Chauvaud, M. Soria (a cura di), Les Vénéneuses. Figures d'empoisonneuses de l'Antiquité à nos jours, Rennes, 2015.

Brambilla 2008 = E. Brambilla, La fine dell'esorcismo: possessione, santità, isteria dall'età barocca all'illuminismo, in Quaderni storici, XXXVIII, 2003, 112, p. 117-164.

Calvi 1981 = G. Calvi, L'oro, il fuoco, le forche, la peste napoletana del 1656, in Archivio Storico Italiano, CXXXIX, 1981-3, p. 406-458.

Calvi 1990 = G. Calvi, "Dell'altrui communicatione»: comportamenti sociali in tempo di peste (Napoli, Roma, Genova 1656-1657), in Popolazione, Società e Ambiente. Temi di Demografia Storica Italiana (secc. XVII-XIX), Bologna, 1990, p. 561-579.
Canneto 2017 = S. Canneto, Santinelli, Francesco Maria, S.v., in Dizionario Biografico degli Italiani, t. 90, Roma, 2017 [on line: http://www.treccani.it/enciclopedia/francesco-maria-santinelli_(Dizionario-Biografico)/].

Cavina 2011 = M. Cavina, Nozze di sangue. Storia della violenza coniugale, Roma-Bari, 2011.

Conforti $2006=$ M. Conforti, Peste a stampa. Trattati, relazioni e cronache a Roma nel 1656, in Roma moderna e contemporanea, XIV-1/3, 2006, p. 135-158.

De Renzi 2008 = S. De Renzi, Per una biografia di Paolo Zacchia: nuovi documenti e ipotesi di ricerca, in Pastore Rossi 2008, p. 50-73.

Di Sivo 2000 = M. Di Sivo, Il fondo della Confraternita di S. Giovanni decollato nell'archivio di Stato di Roma (14971870). Inventario, in Rivista Storica del Lazio, 12, 2000, p. $210-219$.

Donato $2006=$ M.P. Donato, La peste dopo la peste. Economia di un discorso romano (1656-1720), in Fosi 2006, p. 159 174.

Duffin 2011 = J. Duffin, Questioning Medicine in SeventeenthCentury Rome: The consultations of Paolo Zacchia, in Canadian Bulletin of Medical History/Bulletin canadien d'histoire de la Médecine, 28, 201 1, 1, p. 149-170.

Favino 2014 = F. Favino, Pallavicino, Francesco Maria Sforza, s.v., in Dizionario Biografico degli Italiani, t. 80, Roma, 2014 [on line: http://www.treccani.it/enciclopedia/francesco-maria-sforza-pallavicino_(Dizionario-Biografico)/].

Feci 2004 = S. Feci, Pesci fuor d'acqua. Donne a Roma in età moderna: diritti e patrimoni, Roma, 2004.

Fosi 2006 = I. Fosi (a cura di), La peste a Roma (1656-1657), in Roma moderna e contemporanea, XIV, 1-3, 2006.

Fiorani 1978 = L. Fiorani, Astrologi, superstiziosi e devoti nelle società del Seicento, in Ricerche per la storia religiosa a Roma, 2, 1978, p. 97-162.

Fiume 2008 = G. Fiume, Mariti e pidocchi: storia di un processo e di un aceto miracoloso, Roma, 2008.

Forum 2014 = Forum: Donne e poteri nel Rinascimento italiano, in Genesis, 13-2, 2014, p. 169-179.

Kolega 1998 = A. Kolega, Speziali, spagirici, droghieri e ciarlatani, in Roma moderna e contemporanea, VI, 1998, 3, p. 311-347.

La Duca 2016 = R. La Duca, I veleni di Palermo, Palermo, 2016 [ $1^{\text {a }}$ ed. 1970].

Leonardi 2005 = M. Leonardi, Governo, istituzioni, inquisizione nella Sicilia spagnola: i processi per magia e superstizione, Acireale, 2005

Mari - Bertol 2001 = F. Mari, E. Bertol, Veleni. Intrighi e delitti nei secoli, Firenze, 2001.

Marsili 1999 = M. Marsili, Gastaldi, Girolamo, s.v. in Dizionario Biografico degli Italiani, t. 52, Roma, 1999 [on line: http://www.treccani.it/enciclopedia/girolamo-gastaldi_(Dizionario-Biografico)/].

Messana $2007=$ M.S. Messana, Inquisitori, negromanti $e$ streghe nella Sicilia moderna, 1500-1782, Palermo, 2007.

Natale $2008=$ A. Natale, Gli specchi della paura. Il sensazionale e il prodigioso nella letteratura di consumo (secoli XVIIXVIII), Roma, 2008.

Muzzarelli 2013 = M.G. Muzzarelli, Nelle mani delle donne. Nutrire, guarire, avvelenare dal Medioevo a oggi, RomaBari, 2013. 
Orefice 2015 = A. Orefice, I giustiziati di Napoli dal 1556 al 1862 nella documentazione dei Bianchi della Giustizia, Napoli, 2015.

Pallavicino 1837 = S. Pallavicino, Della vita di Alessandro VII, Prato, 1837.

Pallavicino 1839 = S. Pallavicino, Descrizione del contagio che da Napoli si comunicò a Roma nell'anno 1656 e de' saggi provvedimenti ordinati allora da Alessandro VII..., Piacenza, 1839.

Pastore 1990 = A. Pastore, Tra giustizia e politica: il governo della peste a Genova e a Roma nel1656-57, in Popolazione, Società e Ambiente. Temi di Demografia Storica Italiana (secc. XVII-XIX), Bologna, 1990, p. 631-657.

Pastore $1991=\mathrm{A}$. Pastore, Crimine e giustizia in tempo di peste nell'Europa moderna, Roma-Bari, 1991.

Pastore 2001 = A. Pastore, Governare la città appestata: giuristi e medici a confronto, in A. Prosperi (a cura di), Il piacere del testo. Saggi e studi per Albano Biondi, I, Roma, 2001, p. 423-444.

Pastore $2010=$ A. Pastore, Veleno. Credenze, crimini, saperi nell'Italia moderna, Bologna, 2010.

Pastore - Rossi 2008 = A. Pastore, G. Rossi (a cura di), Paolo Zacchia, 1584-1659: alle origini della medicina legale, Milano, 2008.
Preto 1988 = P. Preto, Epidemia, paura e politica nell'Italia moderna, Roma-Bari, 1988.

Rocciolo 2006 = D. Rocciolo, Cum suspicione morbi contagiosi obierunt. Società, religione e peste a Roma nel 1656-1657, in Fosi 2006, p. 111-134.

Salomone-Marino 1881 = S. Salomone-Marino, L'Acqua tofana, in Nuove effemeridi siciliane, s. III, XII, 1881, p. 284-294.

Sonnino 2006 = E. Sonnino, Cronache della peste a Roma. Notizie dal Ghetto e lettere di Girolamo Gastaldi (16561657), in Fosi 2006, p. 35-74.

Sonnino - Traina 1982 = E. Sonnino, R. Traina, La peste del 1656-57 a Roma: organizzazione sanitaria e mortalità, in La demografia storica delle città italiane, Bologna, 1982, p. 433-452.

Vasta 2015-2016 = C. Vasta, Donne criminali. Violenza femminile a Roma, 1550-1630, Università di Roma Tre, Dottorato, a.a. 2015-2016.

Visceglia 2011 = M.A. Visceglia, Attentare al corpo del papa: sortilegi e complotti politici durante il pontificato di Urbano VIII, in V. Lavenia, G. Paolin (a cura di), Riti di passaggio, storie di giustizia. Per Adriano Prosperi, Pisa, 2011. 
